\title{
Pain and Radiographic Outcomes in Adult Idiopathic Scoliosis Patients Using a Scoliosis Activity Suit: An 18-Month Case Controlled Chart Review
}

\author{
Mark W. Morningstar ${ }^{1 *}$, Aatif Siddiqui'2, Clayton J. Stitzel ${ }^{3}$, Brian Dovorany ${ }^{4}$ \\ ${ }^{1}$ Natural Wellness \&Pain Relief Center, Grand Blanc, MI, USA \\ ${ }^{2}$ Esprit Wellness, New York, NY, USA \\ ${ }^{3}$ Lancaster Spinal Health Center, Lititz, PA, USA \\ ${ }^{4}$ Posture \& Spine Care Center, Green Bay, WI, USA \\ Email: ${ }^{*}$ drmorningstar@nwprc.com
}

Received 24 June 2015; accepted 30 August 2015; published 2 September 2015

Copyright (C) 2015 by authors and Scientific Research Publishing Inc.

This work is licensed under the Creative Commons Attribution International License (CC BY). http://creativecommons.org/licenses/by/4.0/

(c) (i) Open Access

\section{Abstract}

There are few conservative treatment options for patients with adult idiopathic scoliosis. These typically include pharmacologic pain management, epidural injections, and generalized CAM treatments such as massage and chiropractic manipulation. The purpose of this study was to compare the post-treatment results in patients wearing the scoliosis activity suit versus baseline assessments as well as adult scoliosis patients who did not wear the activity suit. The pain and Cobb angle outcomes of 53 consecutive patients with adult idiopathic scoliosis following a trial of a scoliosis activity suit were reviewed. The average scores and measurements at 18 months were statistically significantly improved for both the quadruple numerical pain rating scale as well as Cobb angle. The scoliosis activity suit may be a viable supportive therapy for the treatment of chronic pain associated with adult idiopathic scoliosis. Further prospective studies should evaluate treatment effects of this suit using intent-to-treat methodology.

\section{Keywords}

Scoliosis, Pain, Spine, Rehabilitation

\footnotetext{
${ }^{*}$ Corresponding author.
} 


\section{Introduction}

The treatment options for idiopathic scoliosis have greatly developed over the last decade. Bracing techniques [1] and surgical techniques [2] have consistently been made available to adolescent idiopathic scoliosis patients. However, many of the non-surgical procedures have not been available to adult idiopathic scoliosis patients. It is well know that idiopathic scoliosis can continue to progress even into adulthood [3]. When an idiopathic scoliosis measures $>30^{\circ}$ at the time of skeletal maturity, there is approximately a $68 \%$ chance that the curve will continue to progress thereafter [4] [5]. For example, a study by Marty-Poumarat et al. [6], reported a near linear rate of progression year by year into later adulthood.

Most of the treatment studies in adults with idiopathic scoliosis have been surgical. However, common among the various surgical techniques is the incidence of perioperative and postoperative complications, ranging from $17 \%$ - 55\% [7]-[9]. The chance of revision surgery in a study of 306 patients was $44 \%$ in the first 6 years [10]. Since rigid scoliosis bracing is typically indicated for skeletally immature patients, rigid bracing in adulthood is not considered necessary [11]. According to the Society on Scoliosis Orthopedic and Rehabilitation Treatment (SOSORT), bracing for adult curves is indicated only when the therapeutic value of the brace chosen has been established [12]. However, many of the brace technologies established for adult scoliosis patients are European, and their availability in the US is limited at the present time.

With the potential risks of surgical intervention in adulthood, and the limited availability of European bracing technologies in the United States, we report the results of a scoliosis activity suit used in a cohort of patients with adult idiopathic scoliosis.

Previous early data suggest [13] that this suit may help certain pain and radiographic parameters of scoliosis. Here we present our data against a previously published control group with established progression data in adult scoliosis cases.

The scoliosis activity suit is a neoprene, rotation-based activity suit, composed of 4 distinct pieces. The novelty of the scoliosis activity suit is that it creates a rotational resistance into the torso that accentuates the rotational displacement of the scoliosis. This increased rotational stimulus is thought to elicit a corrective postural reflex that results in de-rotation of the spine out of the scoliosis curvature.

\section{Intervention and Outcomes}

Patient files from a single multidisciplinary medical center were consecutively pulled for analysis. All patients had previously reported to this clinic with an established history of adult idiopathic scoliosis. Inclusion criteria for this study included 1) Prior to treatment intervention, all patients completed a quadruple numerical pain rating scale, 2) patients were all skeletally mature at baseline, 3) baseline Cobb angle of at least $30^{\circ}$, and 4) they were not receiving concurrent treatment or being followed up with elsewhere. Patient files were excluded if the type of scoliosis was not idiopathic, as well as patients with history of scoliosis fusion surgery. A total of 53 patient files were consecutively selected, so as to minimize selection bias.

Patients had participated in a fitting process, where the focus was on teaching each patient to properly fit and adjust his/her scoliosis activity suit based upon his/her respective curve pattern. They were also given instruction on which specific activities of daily living to perform while wearing the activity suit. Patients were instructed to wear the activity suit for a total of 4 - 6 hours per day total, in two separate sessions ( 2 - 3 hours each ideally). Figure 1 shows an illustration of a typical configuration for an adult patient.

Once the patients completed their fitting process, they were instructed to report back every 3 months for 1 year, followed by 6 -month intervals thereafter. At each 6-month interval, patients again completed the numerical pain rating scale. These questionnaires were compared to baseline to determine their pain and changes.

The gender breakdown among the 53 total patients selected for this study was 41 females and 12 males. Of these 38 had curves above $30^{\circ}$ while 15 had curves above $50^{\circ}$. When categorized based upon curve pattern, there were 8 patients with thoracolumbar curves, 19 with thoracic curves, 10 with lumbar curves, and 16 with double major curves.

Statistical analysis was performed for the curve pattern groups as well as the baseline Cobb angle groups. Student t-tests were performed to evaluate before and after treatment comparisons. Tables 1-5 provide an outline of this data for the outcome assessments. Across the entire cohort, $79 \%$ of patients achieved a curve correction of $6^{\circ}$ or greater, $17 \%$ had curves unchanged or stabilized (defined as $<6^{\circ}$ of change), and $4 \%$ saw their curves increase. When subdividing the entire cohort by curve pattern, the thoracolumbar group achieved the 


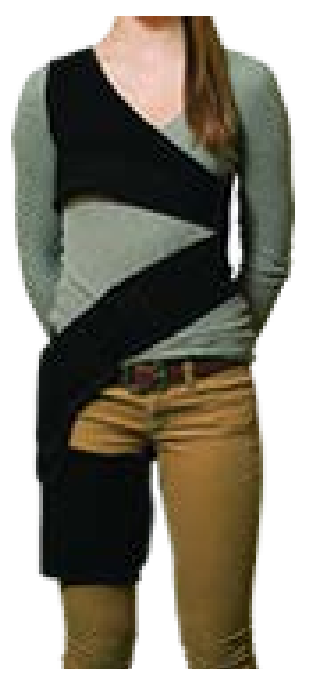

Figure 1. A common sample configuration of the scoliosis activity suit.

Table 1. Entire cohort.

\begin{tabular}{|c|c|c|c|c|c|c|}
\hline Patient & Gender & CurveType & Cobb1 & Cobb2 & QVAS1 & QVAS2 \\
\hline 1 & $\mathrm{f}$ & $\mathrm{L}$ & 55 & 50 & 63 & 43 \\
\hline 2 & $\mathrm{~m}$ & $\mathrm{D}$ & 32 & 22 & 47 & 37 \\
\hline 3 & $\mathrm{f}$ & $\mathrm{L}$ & 36 & 26 & 60 & 53 \\
\hline 4 & $\mathrm{~m}$ & $\mathrm{~T}$ & 52 & 33 & 43 & 33 \\
\hline 5 & $\mathrm{f}$ & $\mathrm{TL}$ & 34 & 30 & 70 & 43 \\
\hline 6 & $\mathrm{f}$ & $\mathrm{T}$ & 42 & 29 & 40 & 20 \\
\hline 7 & $\mathrm{f}$ & $\mathrm{T}$ & 44 & 26 & 30 & 20 \\
\hline 8 & $\mathrm{f}$ & $\mathrm{L}$ & 66 & 40 & 73 & 47 \\
\hline 9 & $\mathrm{~m}$ & $\mathrm{TL}$ & 47 & 30 & 53 & 43 \\
\hline 10 & $\mathrm{f}$ & $\mathrm{D}$ & 33 & 20 & 33 & 30 \\
\hline 11 & $\mathrm{f}$ & $\mathrm{TL}$ & 38 & 30 & 60 & 37 \\
\hline 12 & $\mathrm{~m}$ & $\mathrm{~T}$ & 52 & 35 & 73 & 47 \\
\hline 13 & $\mathrm{f}$ & $\mathrm{L}$ & 61 & 51 & 63 & 47 \\
\hline 14 & $\mathrm{f}$ & $\mathrm{T}$ & 35 & 30 & 33 & 20 \\
\hline 15 & $\mathrm{f}$ & D & 49 & 36 & 37 & 20 \\
\hline 16 & $\mathrm{f}$ & $\mathrm{D}$ & 39 & 33 & 43 & 10 \\
\hline 17 & $\mathrm{f}$ & $\mathrm{L}$ & 40 & 33 & 57 & 30 \\
\hline 18 & $\mathrm{f}$ & $\mathrm{D}$ & 46 & 29 & 43 & 27 \\
\hline 19 & $\mathrm{~m}$ & $\mathrm{~T}$ & 56 & 28 & 57 & 43 \\
\hline 20 & $\mathrm{f}$ & $\mathrm{D}$ & 31 & 20 & 47 & 40 \\
\hline 21 & $\mathrm{f}$ & $\mathrm{T}$ & 38 & 25 & 43 & 30 \\
\hline 22 & $\mathrm{f}$ & $\mathrm{L}$ & 38 & 24 & 67 & 40 \\
\hline
\end{tabular}


M. W. Morningstar et al.

\section{Continued}

\begin{tabular}{|c|c|c|c|c|c|c|}
\hline 23 & $\mathrm{f}$ & $\mathrm{D}$ & 33 & 20 & 37 & 13 \\
\hline 24 & $\mathrm{~m}$ & $\mathrm{D}$ & 32 & 26 & 43 & 27 \\
\hline 25 & $\mathrm{~m}$ & $\mathrm{~T}$ & 30 & 21 & 20 & 20 \\
\hline 26 & $\mathrm{f}$ & $\mathrm{D}$ & 38 & 27 & 33 & 17 \\
\hline 27 & $\mathrm{f}$ & $\mathrm{D}$ & 47 & 33 & 43 & 27 \\
\hline 28 & $\mathrm{f}$ & $\mathrm{T}$ & 41 & 35 & 37 & 30 \\
\hline 29 & $\mathrm{f}$ & $\mathrm{L}$ & 65 & 55 & 60 & 27 \\
\hline 30 & $\mathrm{f}$ & $\mathrm{T}$ & 40 & 18 & 33 & 17 \\
\hline 31 & $\mathrm{f}$ & $\mathrm{T}$ & 54 & 26 & 57 & 27 \\
\hline 32 & $\mathrm{~m}$ & $\mathrm{TL}$ & 39 & 31 & 67 & 23 \\
\hline 33 & $\mathrm{f}$ & $\mathrm{T}$ & 34 & 34 & 43 & 40 \\
\hline 34 & $\mathrm{f}$ & $\mathrm{D}$ & 58 & 59 & 43 & 43 \\
\hline 35 & $\mathrm{f}$ & $\mathrm{T}$ & 37 & 29 & 47 & 40 \\
\hline 36 & $\mathrm{f}$ & $\mathrm{TL}$ & 39 & 20 & 77 & 63 \\
\hline 37 & $\mathrm{f}$ & $\mathrm{L}$ & 53 & 49 & 53 & 43 \\
\hline 38 & f & $\mathrm{T}$ & 44 & 40 & 53 & 47 \\
\hline 39 & $\mathrm{f}$ & $\mathrm{D}$ & 46 & 48 & 60 & 23 \\
\hline 40 & f & $\mathrm{T}$ & 61 & 56 & 73 & 33 \\
\hline 41 & $\mathrm{~m}$ & $\mathrm{D}$ & 45 & 42 & 43 & 40 \\
\hline 42 & $\mathrm{f}$ & $\mathrm{L}$ & 43 & 39 & 50 & 40 \\
\hline 43 & $\mathrm{~m}$ & $\mathrm{~T}$ & 60 & 53 & 60 & 47 \\
\hline 44 & $\mathrm{f}$ & $\mathrm{TL}$ & 48 & 37 & 50 & 37 \\
\hline 45 & $\mathrm{f}$ & $\mathrm{T}$ & 32 & 30 & 37 & 30 \\
\hline 46 & $\mathrm{f}$ & $\mathrm{D}$ & 59 & 52 & 43 & 37 \\
\hline 47 & $\mathrm{f}$ & $\mathrm{L}$ & 39 & 31 & 57 & 33 \\
\hline 48 & $\mathrm{~m}$ & $\mathrm{~T}$ & 53 & 46 & 37 & 20 \\
\hline 49 & $\mathrm{f}$ & $\mathrm{TL}$ & 31 & 15 & 53 & 37 \\
\hline 50 & $\mathrm{f}$ & $\mathrm{D}$ & 30 & 26 & 47 & 30 \\
\hline 51 & $\mathrm{f}$ & $\mathrm{D}$ & 34 & 21 & 53 & 47 \\
\hline 52 & $\mathrm{f}$ & $\mathrm{TL}$ & 32 & 23 & 47 & 40 \\
\hline \multirow[t]{6}{*}{53} & $\mathrm{~m}$ & $\mathrm{~T}$ & 62 & 50 & 67 & 27 \\
\hline & $P$ value & & & $1.36204 \mathrm{E}-06$ & & $1.03956 \mathrm{E}-10$ \\
\hline & Mean & & 43.830189 & 33.43396226 & 50.150943 & 33.67924528 \\
\hline & 42 & $79 \%$ & $>6^{\circ}$ & & & \\
\hline & 9 & $17 \%$ & $<6^{\circ}$ & & & \\
\hline & 2 & $4 \%$ & Progressed & & & \\
\hline
\end{tabular}


M. W. Morningstar et al.

Table 2. Lumbar curves.

\begin{tabular}{|c|c|c|c|c|c|c|}
\hline Patient & Gender & CurveType & Cobb1 & Cobb2 & QVAS1 & QVAS2 \\
\hline 1 & $\mathrm{f}$ & $\mathrm{L}$ & 55 & 50 & 63 & 43 \\
\hline 3 & $\mathrm{f}$ & $\mathrm{L}$ & 36 & 26 & 60 & 53 \\
\hline 8 & $\mathrm{f}$ & $\mathrm{L}$ & 66 & 40 & 73 & 47 \\
\hline 13 & $\mathrm{f}$ & $\mathrm{L}$ & 61 & 51 & 63 & 47 \\
\hline 17 & $\mathrm{f}$ & $\mathrm{L}$ & 40 & 33 & 57 & 30 \\
\hline 22 & $\mathrm{f}$ & $\mathrm{L}$ & 38 & 24 & 67 & 40 \\
\hline 29 & $\mathrm{f}$ & $\mathrm{L}$ & 65 & 55 & 60 & 27 \\
\hline 37 & $\mathrm{f}$ & $\mathrm{L}$ & 53 & 49 & 53 & 43 \\
\hline 42 & $\mathrm{f}$ & $\mathrm{L}$ & 43 & 39 & 50 & 40 \\
\hline \multirow[t]{6}{*}{47} & $\mathrm{f}$ & $\mathrm{L}$ & 39 & 31 & 57 & 33 \\
\hline & & $\mathrm{P}$ value & & 0.035705 & & $6.80694 \mathrm{E}-06$ \\
\hline & & Mean & 49.6 & 39.8 & 60.3 & 40.3 \\
\hline & 7 & $70 \%$ & & & & \\
\hline & 3 & $30 \%$ & & & & \\
\hline & 0 & & & & & \\
\hline
\end{tabular}

Table 3. Double curves.

\begin{tabular}{|c|c|c|c|c|c|c|}
\hline Patient & Gender & CurveType & Cobb1 & Cobb2 & QVAS1 & QVAS2 \\
\hline 23 & $\mathrm{f}$ & D & 33 & 20 & 37 & 13 \\
\hline 24 & $\mathrm{~m}$ & $\mathrm{D}$ & 32 & 26 & 43 & 27 \\
\hline 26 & $\mathrm{f}$ & $\mathrm{D}$ & 38 & 27 & 33 & 17 \\
\hline 27 & $\mathrm{f}$ & $\mathrm{D}$ & 47 & 33 & 43 & 27 \\
\hline 34 & $\mathrm{f}$ & $\mathrm{D}$ & 58 & 59 & 43 & 43 \\
\hline 39 & $\mathrm{f}$ & $\mathrm{D}$ & 46 & 48 & 60 & 23 \\
\hline 41 & $\mathrm{~m}$ & $\mathrm{D}$ & 45 & 42 & 43 & 40 \\
\hline 46 & $\mathrm{f}$ & $\mathrm{D}$ & 59 & 52 & 43 & 37 \\
\hline 50 & $\mathrm{f}$ & $\mathrm{D}$ & 30 & 26 & 47 & 30 \\
\hline \multirow[t]{6}{*}{51} & $\mathrm{f}$ & $\mathrm{D}$ & 34 & 21 & 53 & 47 \\
\hline & & $\mathrm{P}$ value & & 0.01719916 & & $8.47507 \mathrm{E}-05$ \\
\hline & & Mean & 40.75 & 32.125 & 43.4375 & 29.25 \\
\hline & 12 & $75 \%$ & & & & \\
\hline & 2 & $12.50 \%$ & & & & \\
\hline & 2 & $12.50 \%$ & & & & \\
\hline
\end{tabular}


Table 4. Thoracic curves.

\begin{tabular}{|c|c|c|c|c|c|c|}
\hline Patient & Gender & CurveType & Cobb1 & Cobb2 & QVAS1 & QVAS2 \\
\hline 4 & $\mathrm{~m}$ & $\mathrm{~T}$ & 52 & 33 & 43 & 33 \\
\hline 6 & $\mathrm{f}$ & $\mathrm{T}$ & 42 & 29 & 40 & 20 \\
\hline 7 & $\mathrm{f}$ & $\mathrm{T}$ & 44 & 26 & 30 & 20 \\
\hline 12 & $\mathrm{~m}$ & $\mathrm{~T}$ & 52 & 35 & 73 & 47 \\
\hline 14 & $\mathrm{f}$ & $\mathrm{T}$ & 35 & 30 & 33 & 20 \\
\hline 19 & $\mathrm{~m}$ & $\mathrm{~T}$ & 56 & 28 & 57 & 43 \\
\hline 21 & $\mathrm{f}$ & $\mathrm{T}$ & 38 & 25 & 43 & 30 \\
\hline 25 & $\mathrm{~m}$ & $\mathrm{~T}$ & 30 & 21 & 20 & 20 \\
\hline 28 & $\mathrm{f}$ & $\mathrm{T}$ & 41 & 35 & 37 & 30 \\
\hline 30 & $\mathrm{f}$ & $\mathrm{T}$ & 40 & 18 & 33 & 17 \\
\hline 31 & $\mathrm{f}$ & $\mathrm{T}$ & 54 & 26 & 57 & 27 \\
\hline 33 & $\mathrm{f}$ & $\mathrm{T}$ & 34 & 34 & 43 & 40 \\
\hline 35 & $\mathrm{f}$ & $\mathrm{T}$ & 37 & 29 & 47 & 40 \\
\hline 38 & $\mathrm{f}$ & $\mathrm{T}$ & 44 & 40 & 53 & 47 \\
\hline 40 & $\mathrm{f}$ & $\mathrm{T}$ & 61 & 56 & 73 & 33 \\
\hline 43 & $\mathrm{~m}$ & $\mathrm{~T}$ & 60 & 53 & 60 & 47 \\
\hline 45 & f & $\mathrm{T}$ & 32 & 30 & 37 & 30 \\
\hline 48 & $\mathrm{~m}$ & $\mathrm{~T}$ & 53 & 46 & 37 & 20 \\
\hline \multirow[t]{5}{*}{53} & $\mathrm{~m}$ & $\mathrm{~T}$ & 62 & 50 & 67 & 27 \\
\hline & & P Value & & 0.00071025 & & 0.0003936 \\
\hline & & Mean & 45.6315789 & 33.8947368 & 46.4736842 & 31.1052632 \\
\hline & 16 & $84 \%$ & & & & \\
\hline & 3 & $16 \%$ & & & & \\
\hline
\end{tabular}

Table 5. Thoracolumbar curves.

\begin{tabular}{|c|c|c|c|c|c|c|}
\hline Patient & Gender & CurveType & Cobb1 & Cobb2 & QVAS1 & QVAS2 \\
\hline 5 & $\mathrm{f}$ & TL & 34 & 30 & 70 & 43 \\
\hline 9 & $\mathrm{~m}$ & $\mathrm{TL}$ & 47 & 30 & 53 & 43 \\
\hline 11 & $\mathrm{f}$ & TL & 38 & 30 & 60 & 37 \\
\hline 32 & $\mathrm{~m}$ & TL & 39 & 31 & 67 & 23 \\
\hline 36 & $\mathrm{f}$ & TL & 39 & 20 & 77 & 63 \\
\hline 44 & $\mathrm{f}$ & $\mathrm{TL}$ & 48 & 37 & 50 & 37 \\
\hline 49 & $\mathrm{f}$ & $\mathrm{TL}$ & 31 & 15 & 53 & 37 \\
\hline \multirow[t]{5}{*}{52} & $\mathrm{f}$ & $\mathrm{TL}$ & 32 & 23 & 47 & 40 \\
\hline & & P value & & 0.002114 & & 0.00167 \\
\hline & & Mean & 38.5 & 27 & 59.625 & 40.375 \\
\hline & 7 & $88 \%$ & & & & \\
\hline & 1 & $12 \%$ & & & & \\
\hline
\end{tabular}


largest correction, with $88 \%$ of thoracolumbar curves correcting $\geq 6^{\circ}$. The thoracic group achieved this in $84 \%$ of patients. By contrast, the lumbar group saw the lowest correction rate at $70 \%$, followed by the double major group at $75 \%$. However, the double major group is the only group in which patients saw their curves worsen at follow-up (12.5\%). The curve progression in each patient was $1^{\circ}$ and $2^{\circ}$ respectively. This is approximately equal to the natural history of progression for these curves. Figure 2 shows some of the postural changes triggered by application of the suit.

When comparing these curve patterns to the published control group by Marty-Poumarat et al. [6], the average initial age of their subjects was 37 years; ours was 44 . The average starting Cobb angle for lumbar and thoracolumbar curves was $30^{\circ}$, while our lumbar group started at $50^{\circ}$ and our thoracolumbar group was $39^{\circ}$. Thoracic curves began at $44^{\circ}$ for the control subjects, and our thoracic group began with $46^{\circ}$ curves. According to Marty-Poumarat et al.'s data [6], they observed average thoracic curve progression of $0.68^{\circ}$ per year. This would equate to roughly $1.02^{\circ}$ curve increase over our present 18-month study timeframe. Our thoracic group saw their curves improve, by contrast, of $12^{\circ}(\mathrm{P}<0.05)$. Lumbar and thoracolumbar curves increased $1.45^{\circ}$ per year in the control group, whereas our lumbar group saw an average curve correction of $10^{\circ}(\mathrm{P}<0.05)$ and the thoracolumbar group corrected an average $11^{\circ}(\mathrm{P}<0.05)$.

\section{Discussion}

Since most adult scoliosis patients seek therapy for their scoliosis due to pain [12], it is logical to evaluate those methods that seek to accomplish this goal, even if curve correction is unattainable.

Our control group was composed of a previously published cohort by Marty-Poumarat et al. [6]. Our present cohort is similar in demographics and curve characteristics to their cohort, which is why it was chosen. Since Marty-Poumarat et al. provided a detailed, linear rate of progression for adult scoliosis patients, it gave us a realistic comparative by which to evaluate if the scoliosis activity suit could potentially alter the course of natural history of this disease process.

It is important to discuss the limitations of this study. While we did attempt to control the study, it was retrospective in nature, which invariably provides for some degree of selection bias, although we attempted to minimize this by selecting our inclusion criteria before reviewing and selecting patient charts. We also did not perform an intent-to-treat analysis, which would have accounted for the subjects who did not report for followup at 18 months. Future studies should include this analysis.

Although this treatment was primarily performed at home, out present study does not take into account the percentage of compliance within our study population. It is possible that our outcomes could be correlated to the rate of compliance, as well as for those we lost to 18-month follow-up.

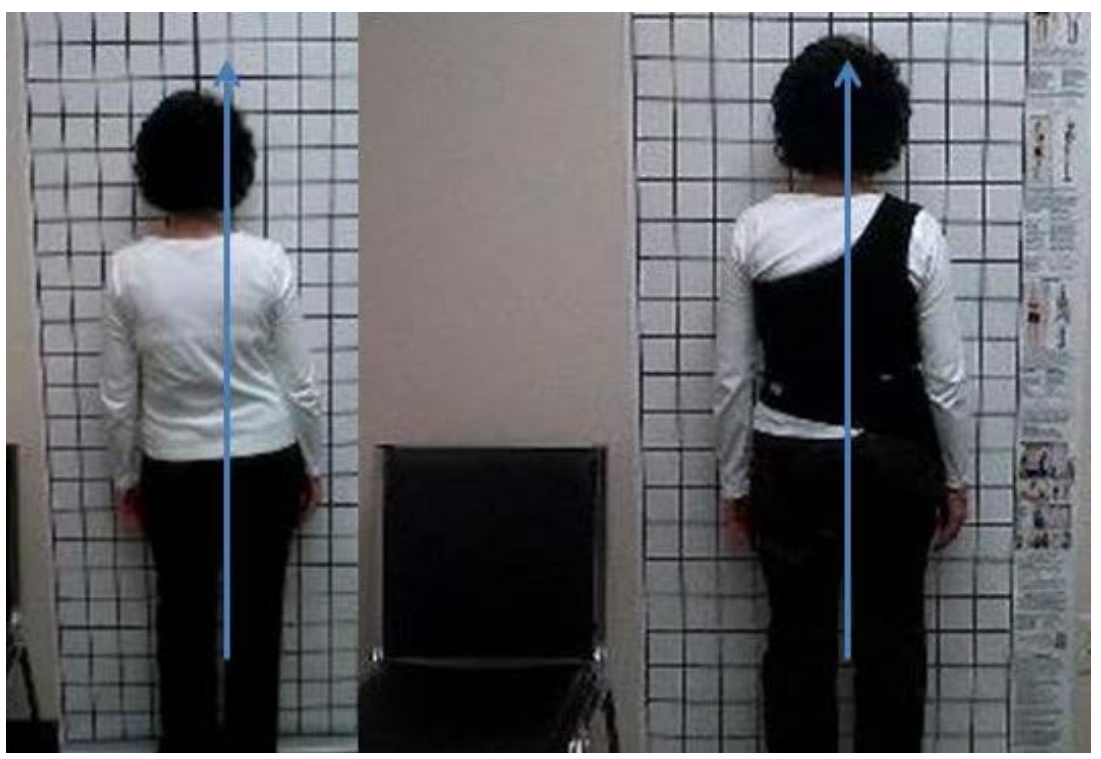

Figure 2. Postural changes associated with the scoliosis activity suit. 
Finally, while pain is a reasonable outcome assessment for this patient population in particular, the quadruple visual analog scale was not specifically designed for scoliosis patients. However, it does provide an easy-tocollect dataset from which to evaluate treatment impact on pain levels. Future studies should include quality of life indices specifically designed for the scoliosis patient population.

\section{Acknowledgements}

The authors declare none.

\section{Conflicts of Interest}

MWM has applied for design and utility patents in the USA on the scoliosis activity suit. The remaining authors declare no conflicts of interest.

\section{References}

[1] Weinstein, S.L., Dolan, L.A., Wright, J.G. and Dobbs, M.B. (2013) Effects of Bracing in Adolescents with Idiopathic Scoliosis. The New England Journal of Medicine, 369, 1512-1521. http://dx.doi.org/10.1056/NEJMoa1307337

[2] Janicki, J.A. and Alman, B. (2007) Scoliosis: Review of Diagnosis and Treatment. Paediatrics \& Child Health, 12, 771-776.

[3] Collis, D.K. and Ponseti, I.V. (1969) Long-Term Follow-Up of Patients with Idiopathic Scoliosis Not Treated Surgically. The Journal of Bone \& Joint Surgery (Am), 51, 425-445.

[4] Weinstein, S.L., Dolan, L.A., Spratt, K.F., Peterson, K.K., Spoonamore, M.J. and Ponseti, I.V. (2003) Health and Function of Patients with Untreated Idiopathic Scoliosis: A 50-Year Natural History Study. JAMA, 289, 559-567. http://dx.doi.org/10.1001/jama.289.5.559

[5] Weinstein, S.L. (1999) Natural history. Spine, 24, 2592-2600. http://dx.doi.org/10.1097/00007632-199912150-00006

[6] Marty-Poumarat, C., Scattin, L., Marpeau, M., Garreau de Loubresse, C. and Aegerter, P. (2007) Natural History of Progressive Adult Scoliosis. Spine, 32, 1227-1234. http://dx.doi.org/10.1097/01.brs.0000263328.89135.a6

[7] Yadla, S., Maltenfort, M.G., Ratliff, J.K. and Harrop, J.S. (2010) Adult Scoliosis Surgery Outcomes: A Systematic Review. Neurosurgical Focus, 28, E3. http://dx.doi.org/10.3171/2009.12.FOCUS09254

[8] Li, G., Passias, P., Kozanek, M., Fu, E., Wang, S., Xia, Q., et al. (2009) Adult Scoliosis in Patients over Sixty-Five Years of Age. Outcomes of Operative versus Nonoperative Treatment at a Minimum Two-Year Follow-Up. Spine, 34, 2165-2170. http://dx.doi.org/10.1097/BRS.0b013e3181b3ff0c

[9] Brodano, B.G., Terzi, S., Gasbarrini, A., Bandiera, S., Simoes, C. and Boriani, S. (2013) Do Benefits Overcome the Risks Related to Surgery for Adult Scoliosis? A Detailed Analysis of a Consecutive Case Series. European Spine Journal, 22, S795-S802. http://dx.doi.org/10.1007/s00586-013-3031-y

[10] Charosky, S., Guigui, P., Blamoutier, A., Roussouly, P. and Chopin, D. (2012) Study Group on Scoliosis. Complications and Risk Factors of Primary Adult Scoliosis Surgery. A Multicenter Study of 306 Patients. Spine, 37, 693-700. http://dx.doi.org/10.1097/BRS.0b013e31822ff5c1

[11] Scoliosis Research Society. https://www.srs.org/patient_and_family/scoliosis/idiopathic/adolescents/bracing.htm

[12] Negrini, S., Aulisa, A.G., Aulisa, L., Circo, A.B., de Mauroy, J.C., Durmala, J., et al. (2012) 2011 SOSORT Guidelines: Orthopaedic and Rehabilitation Treatment of Idiopathic Scoliosis during Growth. Scoliosis, 7, 3. http://dx.doi.org/10.1186/1748-7161-7-3

[13] Morningstar, M. (2013) Outcome Observations in Patients Using a Scoliosis Activity Suit: A Retrospective Chart Review after One-Year Follow-Up. Journal of Scoliosis Rehabilitation, 10, 1-10. 